\title{
Photoelectron Angular Distributions from Liquid Water: Effects of Electron Scattering
}

\author{
Stephan Thürmer, ${ }^{1}$ Robert Seidel, ${ }^{2}$ Manfred Faubel, ${ }^{3}$ Wolfgang Eberhardt,,${ }^{1,4,5}$ John C. Hemminger, ${ }^{6}$ \\ Stephen E. Bradforth, ${ }^{2, *}$ and Bernd Winter ${ }^{1, \dagger}$ \\ ${ }^{1}$ Joint Laboratory for Ultrafast Dynamics in Solutions and at Interfaces, Helmholtz-Zentrum Berlin für Materialien und Energie, \\ Albert-Einstein-Strasse 15, D-12489 Berlin, Germany \\ ${ }^{2}$ Department of Chemistry, University of Southern California, Los Angeles, California 90089, USA \\ ${ }^{3}$ Max-Planck-Institut für Dynamik und Selbstorganisation, Bunsenstrasse 10, D-37073 Göttingen, Germany \\ ${ }^{4}$ Institut für Optik und atomare Physik, TU Berlin, Strasse des 17. Juni 135, D-10623 Berlin, Germany \\ ${ }^{5}$ DESY, Center for Free Electron Laser Science (CFEL), Notkestrasse 85, D-122607 Hamburg, Germany \\ ${ }^{6}$ Department of Chemistry, University of California, Irvine, California 92697, USA
}

(Received 22 November 2012; published 23 October 2013)

\begin{abstract}
Photoelectron angular distributions (PADs) from the liquid-water surface and from bulk liquid water are reported for water oxygen- $1 s$ ionization. Although less so than for the gas phase, the measured PADs from the liquid are remarkably anisotropic, even at electron kinetic energies lower than $100 \mathrm{eV}$, when elastic scattering cross sections for the outgoing electrons with other water molecules are large. The PADs reveal that theoretical estimates of the inelastic mean free path are likely too long at low kinetic energies, and hence the electron probing depth in water, near threshold ionization, appears to be considerably smaller than so far assumed.
\end{abstract}

DOI: 10.1103/PhysRevLett.111.173005

Liquid-microjet photoelectron (PE) spectroscopy marks an important experimental development in accessing electronic structure from highly volatile liquid solutions, particularly the liquid water and aqueous solutions [1]. So far such measurements have aimed at detecting the kinetic energy distribution of the outgoing electrons, $e \mathrm{KE}=h \nu-$ $\mathrm{BE}$, thus enabling determination of values for the electron binding energy (BE). Very little attention has been given, though, to the other primary observable, the photoelectron angular distribution (PAD) from the aqueous solution. For free molecules in the gas phase, PADs reveal information on the orbital character and symmetry from which an electron is ejected $[2,3]$. Thus, with the extension to PAD detection, there is the potential for steady-state and also for newly emerging time-resolved variants of liquid-jet PE spectroscopy [4-7] to access equilibrium molecular orientation and inter-molecular structure in a solution as well as provide a new window on aqueous-phase chemical reactivity. Another motivation for turning attention to the PAD is to resolve an outstanding question as to the probing depth of PE spectroscopy for water [8]. This is of great relevance for depth-profile measurements, which are often applied for identifying the species existing at the surface of atmospheric aerosols $[9,10]$.

There has been an expectation that elastic scattering of the electrons by multiple collisions on the way out of the liquid will wash out any initial anisotropy in the outgoing photoelectrons - to the point that the detected angular distribution will become isotropic. Scattering cross sections for gas-phase water, for which there are extensive compilations of experimental data [11,12] indeed suggest that below $100 \mathrm{eV}$, cross sections for elastic electron scattering are much larger [11] than for inelastic scattering. However,
PACS numbers: $33.20 . \mathrm{Rm}, 33.60 .+\mathrm{q}, 34.80 . \mathrm{Bm}, 79.60 . \mathrm{Cn}$

as the elastic collision cross sections monotonically decrease as electron energy increases, the cross section for inelastic collisions increases markedly as the transfer of energy into dissociation, valence ionization, and electronic excitation become available above $7 \mathrm{eV}$; see also Fig. S1 of the Supplemental Material [13]. This leads to the characteristic maximum of the inelastic scattering cross section, near $100 \mathrm{eV}$. For $>100 \mathrm{eV}, e \mathrm{KE}$ elastic collision cross sections [14] are now comparable with those for inelastic scattering [11]. Various semiempirical and theoretical electron-scattering models have been employed in track simulations for radiation chemistry of liquid water [15-19], but most of these models rely on either gas-phase $[15,16]$ or summed atomic $[18,19]$ collision cross sections for elastic scattering and dielectric theory $[16,17,19]$ for the inelastic processes involving valence electrons. However, there have been no measurements hitherto to assess the quality of the various liquid scattering models.

These very different behaviors of elastic and inelastic scattering probabilities have crucial consequences for photoelectron spectroscopy. Inelastic scattering determines the number of electrons that reach the detector without energy loss. And because of the strong variation of the cross section with energy, this leads to the familiar $e \mathrm{KE}$-dependent probing depth [20], which is connected with the length an electron travels without encountering inelastic collisions (see below). In contrast, the shape of the PAD is the result of elastic scattering - the ratio of elastic-to-inelastic scattering will be the principal determinant of the loss of anisotropy, with respect to the gas-phase anisotropy parameter. We will demonstrate through our PAD measurements that there appear to be discrepancies with most of the scattering models developed for liquid water below $100 \mathrm{eV}$. 
In the present study, we report PAD measurements from liquid water over a wide range of outgoing electron energies. Results will be analyzed using the standard equation for the differential cross section, $d \sigma_{\text {ion }}(h \nu) / d \Omega(\theta)=$ $\sigma_{\text {ion }}(h \nu) / 4 \pi\left[1+\beta(h \nu) P_{2}(\cos \theta)\right][2,21]$, which is known to well apply for gas-phase atoms and randomly oriented molecules. In this expression $\sigma_{\text {ion }}(h \nu)$ is the total photoionization cross section, $\Omega$ is the solid angle of detection, $P_{2}(\cos \theta)=3 / 2 \cos ^{2} \theta-1 / 2$, and $\theta$ is measured with respect to the electric field vector $E$ of the incident light (compare sketch in Fig. 1, left inset). The PAD is thus fully determined by the anisotropy parameter $\beta$, which depends on the subshell and kinetic energy [i.e., $\beta(h \nu)$ through energy conservation becomes $\beta(e \mathrm{KE})]$. These are symmetrical around the light-polarization axis $[8,21]$. Since $d \sigma_{\text {ion }} / d \Omega$ must be positive, $\beta$ ranges from -1 to 2 . For $\beta=0$, the photoemission is isotropic. When introducing an additional symmetry axis, for instance, by orienting molecules upon ordered adsorption on a surface [22], or by aligning gas-phase molecules in a laser field [23], PADs no longer exhibit cylindrical symmetry with respect to the polarization vector. For the water surface, an interesting question is to what extent molecular orientation gets reflected in the measured PAD. Indeed PADs from a single oriented $\mathrm{H}_{2} \mathrm{O}$ (gas), measured in a multicoincidence velocity-map imaging experiment, are highly asymmetric [24], at least for photon energies not too far above the $\mathrm{O} 1 \mathrm{~s}$ ionization threshold.

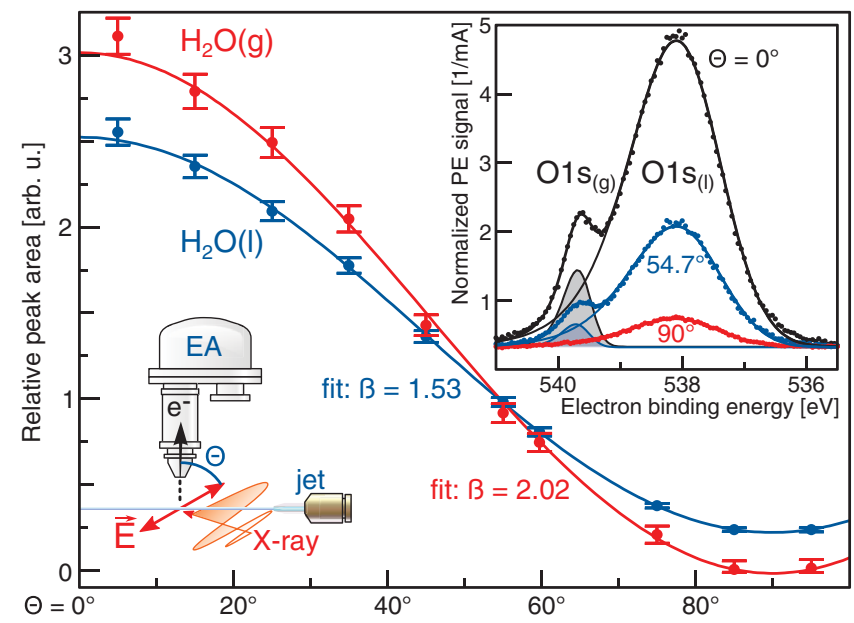

FIG. 1 (color). Right inset: Photon-flux normalized photoelectron spectra for $\mathrm{O} 1 s$ ionization $(h \nu=800 \mathrm{eV})$ at three representative polarization angles $(\theta)$. Solid lines are Gaussian fits to the data. Left inset: Sketch of the experimental geometry. The direction of electron detection by a hemispherical electron analyzer (EA, black arrow), liquid jet, and x-ray beam orthogonally intersect. The light-polarization vector $E$ (red arrow) is varied (change of $\theta$ ) with respect to the EA. Main figure: Peak areas at $800 \mathrm{eV}$ as function of $\theta$, for $\mathrm{H}_{2} \mathrm{O}(\mathrm{l})$ (blue) and $\mathrm{H}_{2} \mathrm{O}(\mathrm{g}$ ) (red). Error bars largely result from experimental reproducibility. Solid lines are $f(\theta)$ fits (see text).
Our PAD measurements are performed for $\mathrm{O} 1 s$ corelevel ionization of $\mathrm{H}_{2} \mathrm{O}$ molecules, both liquid, $\mathrm{H}_{2} \mathrm{O}(\mathrm{l})$, and gas-phase water, $\mathrm{H}_{2} \mathrm{O}$ (gas), simultaneously. The experimental gas-phase $\mathrm{O} 1 s \beta$ values, which surprisingly have not been reported before, provide a crucial reference for interpreting the PADs from liquid water. The reason we chose to focus on core-level ionization is that the oxygen- $1 s$ orbital is localized. In this case strong orbital mixing such as that present for valence orbitals is absent, and also the response to hydrogen bonding is weak, thus placing the focus squarely on the effects of scattering.

The experiments were performed from a $15-\mu \mathrm{m}$ vacuum liquid-water jet (see Ref. [1] for details including sample preparation) using soft $\mathrm{x}$ rays from the UE52-SGM undulator beam line of the BESSY synchrotron facility, Berlin. This beam line provides variable linear polarization directions of the monochromatic x-ray light, and the degree of linear polarization is $100 \%$ at horizontal polarization, dropping to $97 \%$ at vertical polarization. Photon energies were varied between 550 and $1000 \mathrm{eV}$, allowing disentangling of the relative effects of elastic and inelastic scattering on the PAD. Electrons were detected by a hemispherical energy analyzer, in a direction perpendicular to the jet propagation. The jet velocity was approximately $80 \mathrm{~m} \mathrm{~s}^{-1}$, and jet temperature was $5^{\circ} \mathrm{C}$.

Oxygen- $1 s$ photoelectron spectra, simultaneously measured from liquid- and gas-phase water for detection angle $\theta=0^{\circ}$ ( $E \|$ detection), 54.7 (magic angle [21]), and 90 , using $800 \mathrm{eV}$ photon energy, are shown in a further inset of Fig. 1 . The dominant peak at $538.1 \mathrm{eV}$ binding energy is from $\mathrm{H}_{2} \mathrm{O}(\mathrm{l})$, and the smaller $539.7 \mathrm{eV}$ peak is from $\mathrm{H}_{2} \mathrm{O}(\mathrm{g})$ [8]. We quantify the PE signal intensities at this and each different photon energy by fitting the areas of each peak represented by single Gaussians. For spectra measured at a photon energy lower than $600 \mathrm{eV}$, fits account for postcollision interaction effects [25]. Figure 1 presents the relative PE signals obtained as a function of $\theta$. Solid curves are fits to the experimental intensities, where we use $f(\theta)=A\left[1+\beta P_{2}(\cos \theta)\right]$ to find $\beta(e \mathrm{KE})$ which has the above form of $d \sigma_{\text {ion }} / d \Omega(\theta)$. We find that $f(\theta)$ is a good fit to all our experimental data for any of the photon energies used here. This is an important and nontrivial result. It shows that the presence of the surface, despite causing symmetry breaking, is not noticeably reflected in the measured PADs from liquid water.

Figure 2 displays $\beta$ values as a function of energy, obtained from all our O1s PE measurements, for both $\mathrm{H}_{2} \mathrm{O}(\mathrm{g})$ and $\mathrm{H}_{2} \mathrm{O}(\mathrm{l})$. Here, we also include theoretical $\beta$ values for $\mathrm{H}_{2} \mathrm{O}(\mathrm{g})$ [26] for comparison. Notice that scattering off the $\mathrm{H}$ atoms in the initially ionized $\mathrm{H}_{2} \mathrm{O}$ molecule introduces other partial waves $(l \neq 1)$ that will interfere with the outgoing $p$ wave [27], and this leads to $\beta<2$ values in isolated $\mathrm{H}_{2} \mathrm{O}$ for electron energies near the ionization threshold, a result which is experimentally confirmed here. Theory slightly overestimates $\beta$ values, 


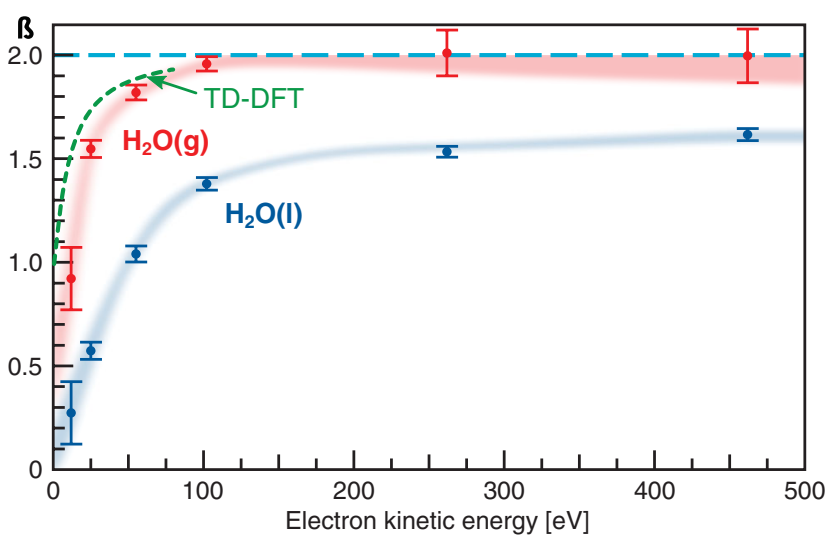

FIG. 2 (color). Experimental $\beta$ values for $\mathrm{O} 1 s$ ionization of $\mathrm{H}_{2} \mathrm{O}(\mathrm{l})$ and $\mathrm{H}_{2} \mathrm{O}(\mathrm{g})$. Error bars are from fitting. Dashed lines are calculated values for $\mathrm{H}_{2} \mathrm{O}(\mathrm{g})$ [26] (green) and for isoelectronic closed-shell $\mathrm{O}^{2-}(\beta=2)$ [2] (cyan) using time-dependent density functional theory (TD-DFT).

though. The most important result from Fig. 2 is that the $\beta$ values for $\mathrm{H}_{2} \mathrm{O}(\mathrm{l})$, although considerably smaller than for gas-phase water, are clearly nonzero, even for $e \mathrm{KEs}$ as low as $10-25 \mathrm{eV}$. The observed anisotropy is an indication that electrons at these energies originate from a fairly small depth into the liquid, as we will detail below.

In order to evaluate the behavior of the experimental $\beta$ values we consider the reduction in anisotropy with respect to $\mathrm{H}_{2} \mathrm{O}(\mathrm{g})$, expressed by $1-\beta_{l} / \beta_{g}$ [which is the percentage drop in $\beta$; see Fig. 3(a), green dots]. A $100 \%$ drop in $\beta$ would correspond to a completely washed out distribution $(\beta=0)$, a result not observed experimentally (Fig. 2). This experimental quantity, and thus the shape of the PAD, is directly connected with the electron elastic-to-inelastic scattering cross-section ratio, or equivalently to the reciprocal ratio of the scattering lengths given by the inelastic mean free path (IMFP) to the elastic one (EMFP) [16]. We can then use such data from the various theoretical models for bulk water, along with a scattering model, to compare the observed energy dependence of $\beta$ against that suggested from current theory. The respective semiempirical IMFP and EMFP curves for liquid water are reproduced in Fig. 3(b). The dark blue line shows the data from Tomita et al. [16], and is representative for a number of similar results from several other authors [17-19]; the fairly small spread of data is quantified by the blue band. In addition, Fig. 3(b) presents the considerably lower IMFP values derived from Pimblott et al. [15] separately. We then use the data from Ref. [15,16] to illustrate theoretical estimates for the IMFP-to-EMFP (equivalent to elastic-to-inelastic scattering) ratio in liquid water in Fig. 3(c). Here, a ratio of, for instance, 100 implies on average 100 elastic collisions before the photoelectron encounters an inelastic scattering event.

To connect these scattering ratios with the experiment [green dots in Fig. 3(a)] we need to quantify electron

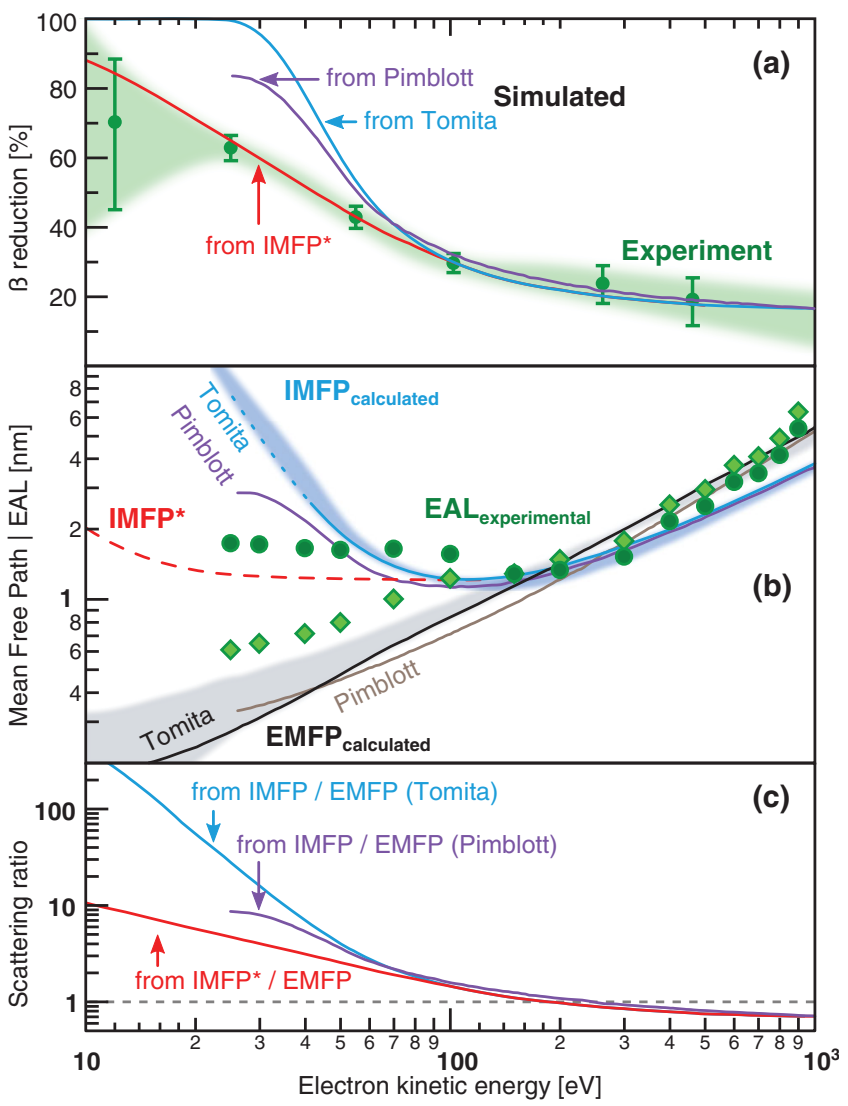

FIG. 3 (color). (a): Experimental (green) and modeled [cyan, violet, red; using IMFP/EMFP ratios from (c)] $\beta$ reduction for $\mathrm{O} 1 s$ ionization of $\mathrm{H}_{2} \mathrm{O}(\mathrm{l})$ relative to $\mathrm{H}_{2} \mathrm{O}(\mathrm{g})$ as a function of $e$ KE. (b): Computed IMFP and EMFP curves for liquid water from Tomita et al. [16] (blue, black) and Pimblott et al. [15] (violet, brown; cross sections were converted to MFP using a density of $\sim 3.35 \times 10^{22}$ molecules $/ \mathrm{cm}^{3}$ ). The shading includes data from other calculations (for IMFP [17,19] and EMFP $[18,19])$. IMFP* (red) is a flattened version for the range $<100 \mathrm{eV}$ (see text). Green symbols are EALs obtained for an aqueous solution taken from Ref. [8], unaltered (circles) and corrected using $\beta$ values of Fig. 2 (diamonds). (c): IMFP/EMFP ratios calculated from the curves of Ref. [16] (blue) and from Ref. [15] (violet), and IMFP*/EMFP (red).

deflection. We will not attempt to precisely model the final electron distribution upon elastic scattering described by a differential elastic collision cross section (DCS), but rather use a mathematical approximation. Let us start by briefly reviewing the shape of DCS for free water molecules; experimental DCS are found, e.g., in Refs. [14,28,29]. Typically two energy regimes are distinguished, below and above $\sim 200 \mathrm{eV} e \mathrm{KE}$ [16]. Below $200 \mathrm{eV}$ one has to consider a fairly complicated DCS including a small chance of backscattering. But here, where multiple scattering is common, we can rather neglect backscattering, and the DCS is expected to exhibit Gaussian-like behavior [30]. Above $200 \mathrm{eV}$, the DCS can be described by Rutherford-like scattering with added screening; but even this situation can be reasonably well described with a 
Gaussian distribution. We thus opt for approximating the DCS over the entire energy range studied here with a Gaussian shape, optimized to represent the experimental data. This is a simple but sufficiently accurate approach for the present purpose. A Gaussian distribution $\exp \left(-\theta^{2} / 2 \varphi^{2}\right)$ with a $\varphi$ of $17^{\circ}$ was modeled according to measurements for gas-phase water [29]. This distribution is used to smear out the initial PAD (decrease of $\beta$ ) successively, i.e., by successive Gaussian convolution where multiple scattering will introduce a factor $n$ to the width. The broadening of the PAD can be qualitatively described by a modified anisotropy parameter $\beta^{*}$, of the form $\beta^{*}(E, \varphi)=$ $\beta(E) \exp \left(-2 n(E) \varphi[\mathrm{rad}]^{2}\right)$. And $n(E)$ is then the average number of elastic scattering events [Fig. 3(c)]. The relative change in $\beta$, in analogy to the corresponding experimental change would then be obtained by calculating $1-\beta^{*} / \beta$, presented by the blue and violet curves in Fig. 3(a). Agreement between experimental and modeled changes of $\beta$ is excellent for $e \mathrm{Ke}>70 \mathrm{eV}$; the observed $20 \%$ base reduction of the $\beta$ value in Fig. 3(a) is assigned to broadening by-on average-one elastic scattering event. However, for the lower energies, experimental values for the change in $\beta$ are $\sim 100$ times smaller than suggested by Ref. [16] and, although in better agreement with the model of Ref. [15], theory still predicts too large a reduction in $\beta$. The large deviation for energies $<70 \mathrm{eV}$ leads to the important result that in most calculations the theoretical IMFP/EMFP ratio is likely overestimated.

Why is the elastic-to-inelastic electron-scattering (or IMFP/EMFP) ratio so much overestimated in theory? It is perhaps not surprising given how closely tied current theoretical models are both to gas-phase scattering cross sections (e.g., for elastic and vibrational inelastic) and the relative paucity of experimental data about electronic excitations and ionization channels in the liquid phase. Differences are in fact fairly pronounced in the electronic structure for bulk water [31] with differences arising from collective excitations and shifts in ionization thresholds from the isolated molecule [32,33]. We argue that it is primarily the IMFP that is overestimated by theory at lower kinetic energies. This is supported by two recent experiments that report on the electron attenuation length (EAL) for electrons ejected from water jets. They suggest the EAL for water in the $<150 \mathrm{eV}$ energy range stays rather flat. Although not directly comparable, the EAL is most closely related to the IMFP, typically being $20 \%$ smaller than IMFP [8,34]. One study is a laser pump-probe PE experiment from an iodide aqueous solution [7], which finds that the technique is surface sensitive even for sub$5 \mathrm{eV} e \mathrm{KE}$. The authors report an effective EAL of $5 \mathrm{~nm}$ for approximately $3 \mathrm{eV}$ electrons, which would be over 100 times smaller than the IMFP values of Tomita et al. [16] A second experiment, from our laboratory [8], estimated the $e$ KE-dependent electron probing depth via the relative water $\mathrm{O} 1 s \mathrm{PE}$ signal as a function of photon energy. The obtained probing depths are presented in Fig. 3(b). Green circles are the original data from Ref. [8] (where constant $\beta$ was assumed for $1 s$ ionization), while green diamonds are the EALs corrected for the new $e$ KE-dependent $\beta$ reported here in Fig. 2, and result in much smaller EALs at low $e \mathrm{KE}$. Adopting the trend suggested by both these experiments, we introduce a flattened IMFP* by decreasing the slope of the $<100 \mathrm{eV}$ part of the IMFP curve of Tomita et al. [16] by factor 100 [yielding the red dashed curve in Fig. 3(b), and the scattering ratio labeled "from IMFP"," Fig. 3(c)]. The resulting $\beta$-reduction curve, shown in red in Fig. 3(a), in fact reproduces rather well our measured PADs (in green). We note that the absolute EAL scale, which, for the study of Ref. [8], was inferred by comparison of PE data from aqueous electrolytes to molecular dynamics simulations of the depth profile of solute ions, is consistent with the range of the reduced IMFP* curve.

In summary, the experimental PADs from liquid water remain noticeably anisotropic even for photon energies merely $10 \mathrm{eV}$ above the $\mathrm{O} 1 s$ ionization threshold, where elastic electron scattering is strong. The reduction of $\beta$ with respect to the values for gas-phase water can be explained quantitatively by the ratio of elastic and inelastic electron-scattering probabilities of the photoelectrons in liquid water. This ratio, while in good agreement for $e \mathrm{KE}>100 \mathrm{eV}$, is distinctly smaller than that predicted by current theoretical models of liquid water at lower kinetic energies, where the IMFP is probably too large. Improved estimates for the importance of inelastic scattering in this energy region can be estimated from the shape of the PADs. Smaller values for the IMFP in liquid water in the $<100 \mathrm{eV}$ energy range are consistent with recent measurements of the electron attenuation length in an aqueous solution, thus supporting the view that PE experiments from liquid water using low-energy electrons (as occurring in both laser UV [5-7] and laser high-harmonic EUV [4]) are actually fairly surface sensitive. Current theoretical models likely do not fully account for the considerable phase dependence for coupling kinetic energy into the various molecular modes, which will lead to different probabilities for accepting the kinetic energy of the electron [15]. For instance, coupling to vibrations, which is determined by the magnitude of the dipole moment, will change because of the considerably larger dipole moment in liquid water [35]. There is also a fundamental difference with regard to the electronic excitation or relaxation mechanisms in the presence of a hydrogen-bonded neighbor water molecule, for example allowing for collective excitations [36] and nonlocal autoionization processes [37]. At present these processes are still poorly understood. The work presented here for liquid water strongly motivates additional studies from the valence electrons of water involved in hydrogen bonding and aimed at revealing PADs from aqueous solutes in different solvation structures at the solution surface or in the bulk. 
The authors thank Albert Stolow and Toshinori Suzuki for stimulating discussions. B. W. and R. S. acknowledge support by the Deutsche Forschungsgemeinschaft (Research Unit FOR 1789, Project No. SE 2253/1-1), and S. E. B. from the U.S. National Science Foundation (Grant No. NSF CHE-0957869).

*stephen.bradforth@usc.edu tbernd.winter@helmholtz-berlin.de

[1] B. Winter and M. Faubel, Chem. Rev. 106, 1176 (2006).

[2] J. Cooper and R. N. Zare, J. Chem. Phys. 48, 942 (1968).

[3] K. L. Reid, Annu. Rev. Phys. Chem. 54, 397 (2003).

[4] K. R. Siefermann, Y.X. Liu, E. Lugovoy, O. Link, M. Faubel, U. Buck, B. Winter, and B. Abel, Nat. Chem. 2, 274 (2010).

[5] A. T. Shreve, T. A. Yen, and D. M. Neumark, Chem. Phys. Lett. 493, 216 (2010).

[6] Y. Tang, H. Shen, K. Sekiguchi, N. Kurahashi, T. Mizuno, Y. I. Suzuki, and T. Suzuki, Phys. Chem. Chem. Phys. 12, 3653 (2010).

[7] F. Buchner, T. Schultz, and A. Lübcke, Phys. Chem. Chem. Phys. 14, 5837 (2012).

[8] N. Ottosson, M. Faubel, S. E. Bradforth, P. Jungwirth, and B. Winter, J. Electron Spectrosc. Relat. Phenom. 177, 60 (2010).

[9] S. Ghosal, J. C. Hemminger, H. Bluhm, B. S. Mun, E. L. D. Hebenstreit, G. Ketteler, D. F. Ogletree, F. G. Requejo, and M. Salmeron, Science 307, 563 (2005).

[10] D. J. Tobias and J. C. Hemminger, Science 319, 1197 (2008).

[11] Y. Itikawa and N. Mason, J. Phys. Chem. Ref. Data 34, 1 (2005).

[12] P. Thorn, M. Brunger, H. Kato, M. Hoshino, and $\mathrm{H}$. Tanaka, J. Phys. B 40, 697 (2007).

[13] See Supplemental Material at http://link.aps.org/ supplemental/10.1103/PhysRevLett.111.173005 for electron collision cross sections.

[14] A. Katase, K. Ishibashi, Y. Matsumoto, T. Sakae, S. Maezono, E. Murakami, K. Watanabe, and H. Maki, J. Phys. B 19, 2715 (1986).

[15] S. M. Pimblott, J. A. LaVerne, and A. Mozumder, J. Phys. Chem. 100, 8595 (1996).

[16] H. Tomita, M. Kai, T. Kusama, and A. Ito, Radiat. Environ. Biophys. 36, 105 (1997).
[17] D. Emfietzoglou and M. Moscovitch, Nucl. Instrum. Methods Phys. Res., Sect. B 193, 71 (2002).

[18] V. A. Semenenko, J. E. Turner, and T. B. Borak, Radiat. Environ. Biophys. 42, 213 (2003).

[19] C. J. Tung, T. C. Chao, H. W. Hsieh, and W. T. Chan, Nucl. Instrum. Methods Phys. Res., Sect. B 262, 231 (2007).

[20] S. Hüfner, Photoelectron Spectroscopy: Principles and Applications (Springer-Verlag, Berlin, 1995).

[21] V. Schmidt, in Electron Spectroscopy of Atoms Using Synchrotron Radiation (Cambridge University Press, Cambridge, England, 1997).

[22] E. W. Plummer and W. Eberhardt, Adv. Chem. Phys. 49, 533 (1982).

[23] J. G. Underwood and K. L. Reid, J. Chem. Phys. 113, 1067 (2000).

[24] M. Yamazaki, J. I. Adachi, T. Teramoto, A. Yagishita, M. Stener, and P. Decleva, J. Phys. B 42, 051001 (2009).

[25] P. van der Straten, R. Morgenstern, and A. Niehaus, Z. Phys. D 8, 35 (1988).

[26] M. Stener, G. Fronzoni, D. Toffoli, and P. Decleva, Chem. Phys. 282, 337 (2002).

[27] S. T. Manson and A. F. Starace, Rev. Mod. Phys. 54, 389 (1982).

[28] M. A. Khakoo, H. Silva, J. Muse, M. C. A. Lopes, C. Winstead, and V. McKoy, Phys. Rev. A 78, 052710 (2008).

[29] H. Cho, Y. S. Park, H. Tanaka, and S. J. Buckman, J. Phys. B 37, 625 (2004).

[30] S. Goudsmit and J. L. Saunderson, Phys. Rev. 57, 24 (1940).

[31] H. Hayashi, N. Watanabe, Y. Udagawa, and C.-C. Kao, Proc. Natl. Acad. Sci. U.S.A. 97, 6264 (2000).

[32] B. Winter, R. Weber, W. Widdra, M. Dittmar, M. Faubel, and I. V. Hertel, J. Phys. Chem. A 108, 2625 (2004).

[33] C. G. Elles, A.E. Jailaubekov, R. A. Crowell, and S.E. Bradforth, J. Chem. Phys. 125, 044515 (2006).

[34] A. Jablonski and C. Powell, J. Electron Spectrosc. Relat. Phenom. 100, 137 (1999).

[35] P. L. Silvestrelli and M. Parrinello, Phys. Rev. Lett. 82, 3308 (1999).

[36] C. D. Wilson, C. A. Dukes, and R. A. Baragiola, Phys. Rev. B 63, 121101 (2001).

[37] S. Thürmer, M. Ončák, N. Ottosson, R. Seidel, U. Hergenhahn, S. E. Bradforth, P. Slavíček, and B. Winter, Nat. Chem. 5, 590 (2013). 